Nonlinear Processes in Geophysics, 12, 955-964, 2005

SRef-ID: $1607-7946 / \mathrm{npg} / 2005-12-955$

European Geosciences Union

(C) 2005 Author(s). This work is licensed

under a Creative Commons License.

\title{
Numerical modelling of disintegration of basin-scale internal waves in a tank filled with stratified water
}

\author{
N. Stashchuk ${ }^{1}$, V. Vlasenko ${ }^{1}$, and K. Hutter ${ }^{2}$ \\ ${ }^{1}$ School of Earth, Ocean and Environmental Sciences, Plymouth University, Drake Circus, Plymouth PL4 8AA, UK \\ ${ }^{2}$ Institute of Mechanics, Darmstadt University of Technology, Hochschulstr. 1, 64289 Darmstadt, Germany \\ Received: 3 August 2005 - Revised: 28 October 2005 - Accepted: 28 October 2005 - Published: 9 November 2005 \\ Part of Special Issue "Turbulent transport in geosciences"
}

\begin{abstract}
We present the results of numerical experiments performed with the use of a fully non-linear non-hydrostatic numerical model to study the baroclinic response of a long narrow tank filled with stratified water to an initially tilted interface. Upon release, the system starts to oscillate with an eigen frequency corresponding to basin-scale baroclinic gravitational seiches. Field observations suggest that the disintegration of basin-scale internal waves into packets of solitary waves, shear instabilities, billows and spots of mixed water are important mechanisms for the transfer of energy within stratified lakes. Laboratory experiments performed by D. A. Horn, J. Imberger and G. N. Ivey (JFM, 2001) reproduced several regimes, which include damped linear waves and solitary waves. The generation of billows and shear instabilities induced by the basin-scale wave was, however, not sufficiently studied.

The developed numerical model computes a variety of flows, which were not observed with the experimental set-up. In particular, the model results showed that under conditions of low dissipation, the regimes of billows and supercritical flows may transform into a solitary wave regime. The obtained results can help in the interpretation of numerous observations of mixing processes in real lakes.
\end{abstract}

\section{Introduction}

For lakes with trench-like profiles, measurements have shown that the pycnocline changes after wind events. The connection of these processes with internal seiches was reported by Mortimer (1952) for the long and narrow Windermere (UK) and for Loch Ness (UK) by Thorpe (1977). More recent observations of basin-scale and higher-frequency internal waves after wind events were reported by Stevens (1999) for the Sook Lake Reservoir near Victoria, British Columbia, Canada.

Correspondence to: N. Stashchuk

(nstashchuk@plymouth.ac.uk)
The baroclinic response of a stratified lake to wind forcing depends on the shape of the shore line, bottom topography, water stratification, and intensity and direction of the wind. Measurements by Thorpe (1974) in Loch Ness, for example, had shown that the generated baroclinic seiche disintegrates into shorter waves during its oscillation.

The study of the disintegration of a baroclinic seiche into short period internal waves can be simplified by consideration of this process in a rectangular tank. In such a case all secondary effects will be removed. The wind blowing along the lake upwells the pycnocline in one part of the lake and downwells it at the opposite side. The inclination of the pycnocline in the tank may be considered to be a result of wind action.

Horn et al. (2001), with the help of a simple two-layer rectangular model found five different wave regimes of the disintegration of the initial basin-scale internal seiche into small-scale internal waves: (i) damped linear waves; (ii) solitons; (iii) supercritical flow; (iv) Kelvin-Helmholz billows; (v) bores and billows. The names of regimes were given according to the basic phenomenon which predominates at the beginning of the motion. They found that for the long narrow tank filled with stratified water the baroclinic wave regime depends basically upon two nondimensional parameters: the undisturbed position of the interface, $h / H$, defined as the ratio of the pycnocline depth $h$ to the overall tank depth $H$, and the non-dimensional amplitude of the initial basin-scale wave defined as the ratio of the initial deflection of the pycnocline $\eta$ to its undisturbed depth $h$ (Fig. 1).

Taking into account that the period of an internal seiche in an enclosed basin is given by equation

$T=2 D / c_{p}$,

the seiche flow is periodic with period $T$ and maximum velocities occurring when the flow is horizontal at $t=T / 4,3 T / 4,5 T / 4, \ldots$ Here $D$ is the length of the tank, $c_{p}$ the linear long-wave phase speed

$c_{p}=\left[g^{\prime} h(H-h) / H\right]^{1 / 2}$, 


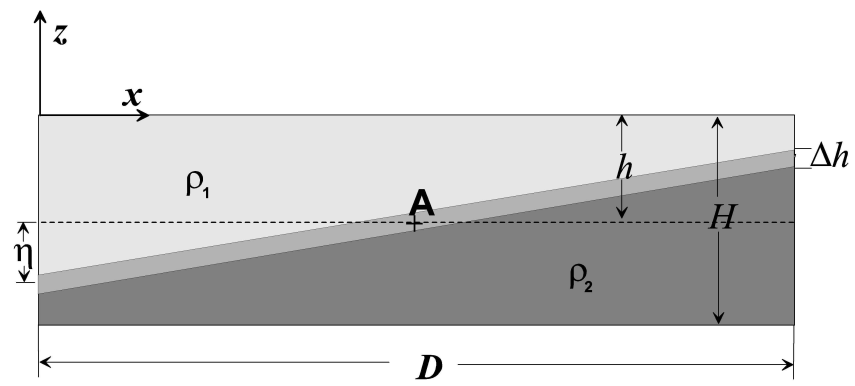

Fig. 1. Schematic diagram of the model set-up.

where $g^{\prime}=\left(\Delta \rho / \rho_{0}\right) g, g$ is the acceleration due to gravity, $\rho_{0}$ the averaged density and $\Delta \rho$ the density difference between the two layers.

When $t=T / 4$, horizontal velocities at the centre of the basin become maximal and the development of KelvinHelmholtz instabilities is one of the possible scenarios for the flow evolution. The formation of Kelvin-Helmholtz billows depends on the value of the local Richardson number,

$R i=\frac{g^{\prime} \Delta h}{(\Delta U)^{2}}$

and it is probable when $R i$ attains the critical value. Here $\Delta h$ is the thickness of the density interface and $\Delta U=g^{\prime} 2 \eta t / D$ (Horn et al., 2001) is the difference between the velocities of the layers. If the value of $R i=0.25$ is considered as critical for the development of instabilities, the flow is stable for times $t<T_{K H}$, where

$T_{K H}=\frac{D}{\eta}\left(\frac{\Delta h}{g^{\prime}}\right)^{1 / 2}$.

The flow in the tank will remain stable if $T_{K H} \geq T / 4$ where $T$ is given by Eq. (1).

There is also an additional important process which can develop in a tank at large initial inclination of the interface. Being strongly inclined the density interface begins to move faster than the phase velocity of long internal waves $c_{p}$. This gives rise to a supercritical regime, and the local Froude number becomes larger than unity. For a two-layer fluid the Froude number can be introduced as

$F=\left(F_{1}^{2}+F_{2}^{2}\right)^{1 / 2}$,

where $F_{1}^{2}=U_{1}^{2} / g^{\prime} h, F_{2}^{2}=U_{2}^{2} / g^{\prime}(H-h), U_{1}$ and $U_{2}$ are the velocities in the upper and lower layers, respectively. The time at which the flow becomes first supercritical by setting $F=1$ is given by (Horn et al., 2001)

$T_{b}=\frac{T}{4} \frac{h}{\eta}\left[\frac{H(H-h)^{2}}{h^{3}+(H-h)^{3}}\right]^{1 / 2}$.

If $T_{b}<T / 4$ the flow becomes supercritical.

The non-linear steepening of the density interface is the next important process which results in the formation of the bore and its disintegration into solitary waves. According to a weakly nonlinear theory solitary waves can be explained by the Korteweg-de Vries equation

$\zeta_{t}+c_{p} \zeta_{x}+\alpha \zeta \zeta_{x}+\beta \zeta_{x x x}=0$

where $\zeta$ is the wave displacement, and $\alpha$ and $\beta$ are the nonlinear and dispersion coefficients, respectively. For a twolayer fluid these coefficients read

$\alpha=\frac{3 c_{p}(H-2 h)}{2(H-h) h}, \quad \beta=c_{p} \frac{(H-h) h}{6}$.

Equation (7) can be rewritten in terms of characteristic lines, viz. $d x / d t=-\left(c_{p}+\alpha \zeta\right)$ when the dispersion term is neglected. It is seen that the lineaer phase speed $c_{p}$ is corrected by the term $\alpha \zeta$, which is responsible for the wave steepening. Thus, the steepening timescale can be simply determined as

$T_{s} \sim \frac{D}{\alpha \eta}$.

If $T_{s}>T_{d}$, where $T_{d}$ is the time of viscous damping,

$T_{d}=\frac{1}{\gamma}$,

the viscosity supresses the formation of solitary waves and the internal seiche attenuates gradually with time. Here $\gamma$ is the measure of the e-foding energy decay. To find $\gamma$ we follow Batchelor (1967) who estimated the rate of energy dissipation in the boundary layer per unit area, $\tilde{E}_{t}$, as

$\frac{d \tilde{E}}{d t}=-\frac{\rho_{0} \nu U^{2}}{2 \delta}$.

Here $U$ is the maximum flow velocity outside the boundary layer, $\delta=(\nu T / \pi)^{1 / 2}$ is its thickness, and $\nu$ is the viscosity. The total energy dissipation rate in the top, bottom and interface boundary layers can be expessed according to Eq. (11) as

$\frac{d E}{d t}=-\int_{A}\left[\frac{\rho_{0} v U_{1}^{2}}{2 \delta}+\frac{\rho_{0} v U_{2}^{2}}{2 \delta}+\frac{\rho_{0} v \Delta U^{2}}{2 \Delta h}\right] d A$,

where $\Delta U=U_{1}-U_{2}$ is velocity difference between layers and $A$ is the area of the boundary layer. Taking into account that $U_{j}(x)=\hat{U}_{j}(D-|D-2 x|) / D(j=1,2)$ and $U_{1} h=-U_{2}(H-h)$, Eq. (12) can be rewritten as

$\frac{d E}{d t}=-\frac{v \rho_{0} A \hat{U}_{2}^{2}}{6}\left[\frac{1}{\delta}\left(\frac{H-h}{h}\right)^{2}+\frac{1}{\Delta h}\left(\frac{H}{h}\right)^{2}\right]$.

The total energy of the internal seiche is given by

$E=\frac{\rho_{0} A}{6}\left[\hat{U}_{1}^{2} h+\hat{U}_{2}^{2}(H-h)\right]=\frac{\rho_{0} A}{6} \frac{(H-h) H}{h} \hat{U}_{2}^{2}$.

The ratio of Eqs. (13) to (14) gives the coefficient of the energy decay

$\gamma=\frac{v}{\delta} \frac{(H-h)}{h H}+\frac{v}{\Delta h} \frac{H}{h(H-h)}$. 
The times cales presented above are based on the theory valid for weakly non-linear and two layer fluid approximations. In the present paper we suggest an approach, which is free of the disadvantages of two-layer models and which can incorporate effects of strong nonlinearity and nonhydrostaticity. We apply the mathematical numerical model developed on a very fine grid to examine the influence of nonlinearity of a wave process on the mechanism of energy transfer from large to small scale motions (and eventually to turbulence and mixing, as an extreme situation).

\section{Mathematical model}

The water motion in a tank is considered in the Cartesian coordinate system $0 x y z$ with the $\mathrm{z}$-axis directed upward $(z=0$ is the top boundary of the tank), $\mathrm{x}$-axis is directed along the tank, and $\mathrm{y}$-axis is perpendicular to the $(x, z)$ plane (Fig. 1). In the two-dimensional case when the transverse motions are neglected, the governing system of equations reads

$$
\begin{aligned}
& u_{t}+u u_{x}+w u_{z}=-P_{x} / \bar{\rho}_{0}+v\left(u_{x x}+u_{z z}\right), \\
& w_{t}+u w_{x}+w w_{z}=-P_{z} / \bar{\rho}_{0}-g \rho / \overline{\rho_{0}}+v\left(w_{x x}+w_{z z}\right), \\
& u_{x}+w_{z}=0, \\
& \rho_{t}+u \rho_{x}+w \rho_{z}-\overline{\rho_{0}} / g N^{2}(z) w=k\left(\rho_{x x}+\rho_{z z}\right) .
\end{aligned}
$$

Here, $u$ and $w$ are the velocities of the water in the $x$ and $z$ directions; $P$ and $\rho$ are the pressure and the density respectively; $\overline{\rho_{0}}=$ const is the reference density, $v$ the coefficient of viscosity, and $k$ the coefficient of diffusivity and $N(z)=\left(-g \rho_{0 z} / \bar{\rho}_{0}\right)^{1 / 2}$ is the buoyancy frequency.

Introducing the stream function $\psi\left(u=\psi_{z}, w=-\psi_{x}\right)$ and the vorticity $\omega\left(\omega=\psi_{x x}+\psi_{z z}\right)$, system (Eq. 16) can be rewritten as

$$
\begin{aligned}
& \omega_{t}+J(\omega, \psi)=g \rho_{x} / \overline{\rho_{0}}+v\left(\omega_{x x}+\omega_{z z}\right), \\
& \rho_{t}+J(\rho, \psi)+\bar{\rho}_{0} / g N^{2}(z) \psi_{x}=k\left(\rho_{x x}+\rho_{z z}\right),
\end{aligned}
$$

where $J(A, B)=A_{x} B_{z}-A_{z} B_{x}$ is the Jacobian operator.

The following boundary conditions are used at the top, $z=0$, and bottom, $z=-H$, of the tank:

$\psi=0, \quad \psi_{z}=0, \quad \rho_{z}=0, \quad \omega=\omega_{0}$,

and at the lateral left, $x=0$, and right, $x=D$, boundaries

$\psi=0, \quad \psi_{x}=0, \quad \rho_{x}=0, \quad \omega=\omega_{0}$.

Here the value of $\omega_{0} \neq$ const is calculated from the previous temporal step with $\omega_{0}=0$ when $t=0$.

The problem (Eqs. 17-19) is solved numerically with the help of the alternative direction implicit method (Vlasenko et al., 2005). The numerical runs were carried out with the molecular value of viscosity $\nu=10^{-6} \mathrm{~m}^{2} \mathrm{~s}^{-1}$, and with diffusivity $k=10^{-6} \mathrm{~m}^{2} \mathrm{~s}^{-1}$. The spatial resolution was $\Delta x=\Delta z=2.4 \times 10^{-4} \mathrm{~m}$. Stability of the model scheme was achieved by taking $\Delta t=10^{-2} \mathrm{~s}$.

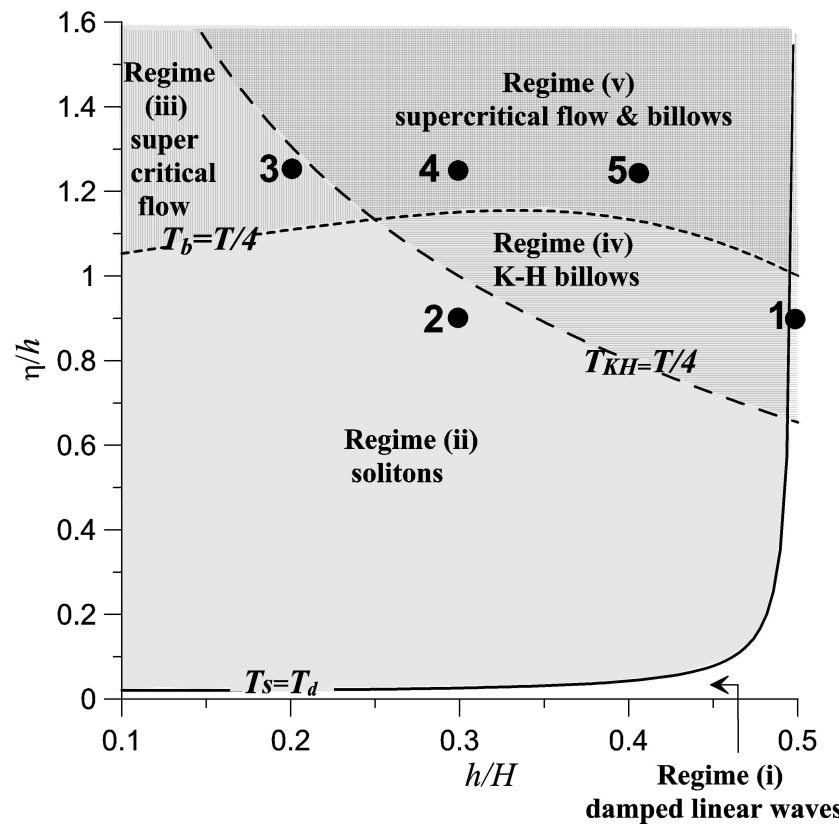

Fig. 2. The regime boundaries for the basin-scale seiche disintegration, plotted in terms of the initial basin-scale wave $\eta / h$ and the depth ratio $h / H$ when $H=0.29 \mathrm{~m}, D=6 \mathrm{~m}, \Delta \rho=20 \mathrm{~kg} \mathrm{~m}^{-3}$, $\Delta h=0.02 \mathrm{~m}$. The cases discussed here are identified by the numbered dots.

\section{Scenarios of seiche evolution}

The main parameters of the experimental equipment of Horn et al. (2001) were used as a basis for the modelling. The fully enclosed experimental tank, $600 \mathrm{~m}$ long $(D), 0.29 \mathrm{~m}$ deep $(H)$ and $0.30 \mathrm{~m}$ wide (Fig. 1) was replaced by a mathematical region infinite in the $y$-direction and the same values of length $D=600 \mathrm{~m}$ and depth $H=0.29 \mathrm{~m}$. The density contrast between the layers, $\Delta \rho=20 \mathrm{~kg} \mathrm{~m}^{-3}$, and the layer thickness $\Delta h=0.02 \mathrm{~m}$ was similar to that in the laboratory tank.

In the laboratory experiments the interface was generally below the mid-depth position so that the lower layer was the thinner layer. This is the reverse of the stratification found in most lakes in which the surface layer is usually thinner than the lower layer. Because of the rigid lid and constant depth of the tank, this reversal does not alter the physics of the system in the laboratory experiments.

Figure 2 shows the boundaries between different regimes obtained in the $(\eta / h, h / H)$ plane. The thick line $T_{s}=T_{d}$ separates the regimes of solitons and damped linear waves. Taking into account Eqs. (8)-(10) and (15) we can expect generation of solitary waves when

$$
\frac{\eta}{h}>\frac{2 v D}{3 H c_{p}} \frac{\left[\left(\pi c_{p} / 2 v D\right)^{1 / 2}(1-h / H)^{2}+1 / \Delta h\right]}{h / H(1-2 h / H)} .
$$

If the Froude number equals unity supercritical flow precedes the formation of solitons. The boundary $T_{b}=T / 4$ of 


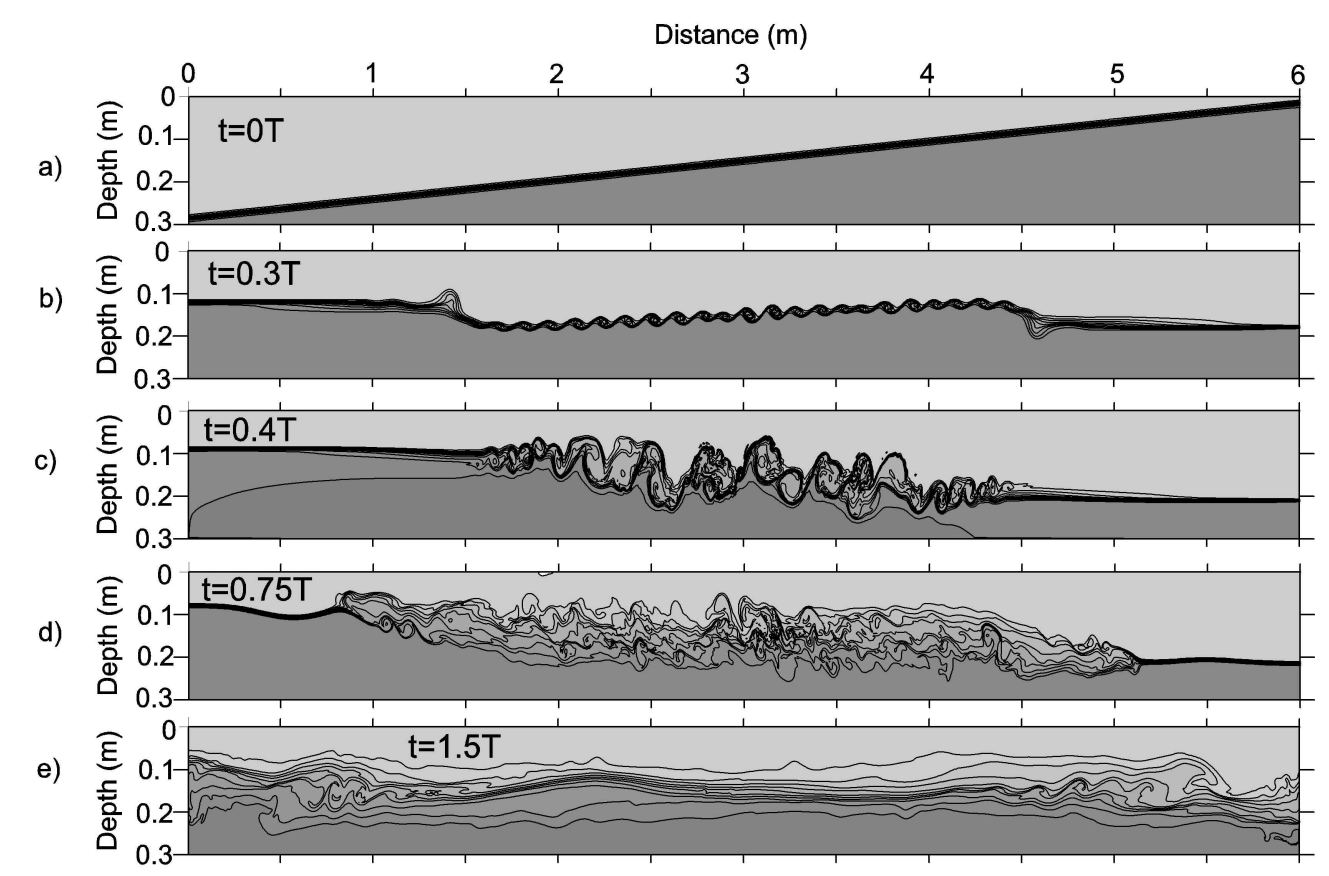

Fig. 3. Evolution of the density field for case 1 (Fig. 2), at $h / H=0.5, \eta / h=0.9$ (regime "Kelvin-Helmholtz billows").

this regime is given by the dotted line in Fig. 2, and supercritical flow is formed under the conditions

$\frac{\eta}{h}>\left[\frac{(1-h / H)^{2}}{(h / H)^{3}+(1-h / H)^{3}}\right]^{1 / 2}$.

This equation was obtained with the help of Eq. (1) and Eq. (6).

The dashed line $T_{K H}=T / 4$ separates regimes II and III from IV and V when the shear between layers becomes large enough and the Richardson number falls to 1/4. KelvinHelmholtz billows are formed when

$\frac{\eta}{h}>2\left[\left(\frac{1}{h / H}-1\right) \frac{\Delta h}{H}\right]^{1 / 2}$.

Thus three curves in Fig. 2 divide the $(\eta / h, h / H)$ plane into five regimes: (i) damped linear waves, (ii) solitons, (iii) supercritical flow, (iv) Kelvin-Helmholz billows and (v) supercritical flow and billows. Inspection of Eqs. (20)-(22) shows that only the dotted curve $T_{b}=T / 4$ which is the boundary between subcritical and supercritical conditions is independent of the fluid stratification and dimensions of the tank.

With the help of the numerical model we reproduce all regimes presented in Fig. 2. Results of five experimemts marked by circle with numbers are discussed below.

\subsection{Regime (iv): Kelvin-Helmholtz billows}

We start our analysis with the consideration of the regime "Kelvin-Helmholtz billows" to point out some important features of the performed analysis.

In many fluid systems, turbulence and mixing are governed by a competition between large scale shear and stable ambient density stratification. The Kelvin-Helmholtz instability of the stratified, parallel shear layer has been a standard model for this class of flows ever since it was described by Kelvin in 1871.

A simple way to produce Kelvin-Helmholtz billows under laboratory conditions was suggested by Thorpe (1971, 1973). The methodology of the experiments was similar to that of Horn et al. (2001): an inclined interface of the waters with different densities was produced by a rotation of the tank. After sudden back-rotation the ensuing evolution of the interface produces several types of motion described above. The trick to avoid the generation of internal solitary waves is to arrange the initial conditions at $t=0$ in such a way that the initial density interface coincides with the tank's diagonal. As is known from the theory, the quadratic term $\alpha$ in a weakly nonlinear Korteweg-de Vries Eq. (7) vanishes when the interface is located exactly in the middle of the water depth.

More evidently, the absence of solitary waves can be seen from a dependence of the wavelength $\lambda$ upon the amplitude of solitary wave, $a$. For a two-layer fluid, as in the considered case, the relationship $\lambda=\lambda(a)$ reads

$\lambda^{2}=\frac{4}{3} \frac{h^{2}(H-h)^{2}}{a(H-2 h)}$

(Ostrovsky and Stepanynts , 1989). From this equation it is evident that $\lambda \rightarrow \infty$ when $h \rightarrow H / 2$.

Thus, the disintegration of a basin-scale internal wave into a series of solitary waves becomes impossible, and generation of only Kelvin-Helmholtz billows is expected. Of course, this process occurs only in the presence of strong 


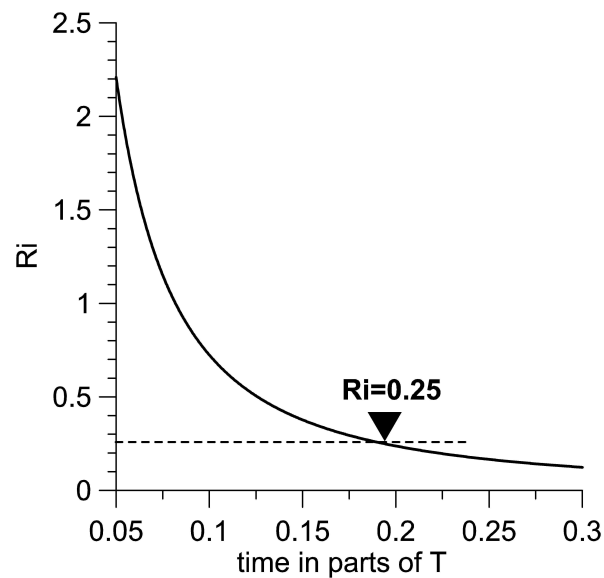

Fig. 4. The dependence of the value of Richardson number vs time for the regime "Kelvin-Helmholtz" billows.

shearing currents when $R i<0.25$ (Turner, 1973) and the flow loses its stability.

An example of the reconstruction of the background density profile due to mixing produced by billows is shown in Fig. 3. The initial parameters for this run were: $h=0.145$ $\mathrm{m}$ (the interface lies exactly in the middle between surface and bottom) and $\eta=0.1305 \mathrm{~m}$. The dimensionless values are $h / H=0.5, \eta / h=0.9$.

Two bores are generated at the very beginning of the motion near the left and right walls. These bores move to the centre of the tank. During their propagation an intensification ("compression") of the motion in the central part of the tank leads to an increase of the horizontal velocity shear. When $t=0.3 T$ is reached, the Richardson number falls below the critical value, and the process of generation of billows due to the Kelvin-Helmholtz instability is initiated.

The value of the local Richardson number (Eq. 3) in the central part of the tank (point A, see Fig. 1) for different time slices is shown in Fig. 4. When $t=0.2 T$ the Richardson number $R i$ reaches its "critical" value 0.25 , but the water motion is still stable. The development of rolls of fluid due to instability begins at $t=0.3 T$ when $R i$ has dropped to the value 0.125 .

The more detailed structure of the density field with billows (zoom panel at $t=0.3 T$ ) is shown in Fig. 5. The billows grow in amplitude and lose their regular structure as the bores continue their propagation to the centre of the basin. Gradually, the wave motions transform from the laminar to the turbulent form. Manifestation of this process can be seen by a comparison of the density field at the time interval $t=0.3-0.4 T$ (Fig. 3). This process of pycnocline erosion continues as long as essential turbulent pulsations exist within the interface area. The turbulence gradually attenuates with time and, finally, the density field takes a form similar to that presented in Fig. 3e. Instead of the initial sharp interface, the vertical density profile has been washed out. The light and heavy waters are mixed and the pycnocline thickness becomes ten times larger at $t=1.5 T$ than it was at the very beginning of the seiche oscillations.

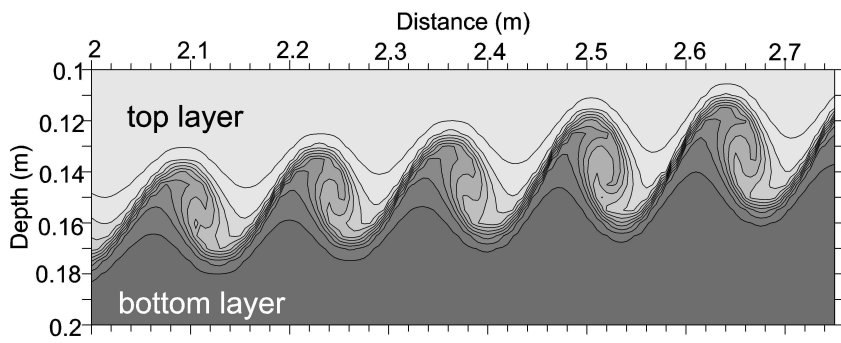

Fig. 5. Enlarged part of the density field presented in Fig. 3b with eddies $(t=0.3 T)$.

The correct numerical modelling of the Kelvin-Helmholtz instability and successive transformation of the initially generated billows into homogeneous turbulence and its final attenuation is possible only with the use of a very fine grid. To check the performance of the numerical grid used in calculations let us consider some remarks on this topic based on the ideas of Kolmogorov's theory of turbulence (Kolmogorov, 1941)

The turbulent density field can be thought of as being made of many eddies of different sizes. Energy must be fed at some rate $\varepsilon$ per unit mass per unit time at the largest eddies of size $L$ and velocity $U$, for which the Reynolds number is $R e=L U / v \gg 1$. The energy then cascades to smaller eddies until it reaches eddies with size $l_{s}$ and velocity $u_{s}$ for which $R e \sim 1$, and the energy in these eddies is lost to viscous dissipation.

Energy is not built up at any smaller scale; the intermediate eddies transmit energy $\varepsilon$ to the smaller eddies. Kolmogorov postulated that it must be possible to express $\varepsilon$ in terms of scales $l$ and $u$, inherent to the smallest eddies, and on dimensional grounds the only way of writing $\varepsilon$ in terms of $l$ and $u$ is $\varepsilon \sim u^{3} / l$. Now it is easy to find that $L / l_{s} \sim R e^{3 / 4}$; in other words, the Reynolds number associated with the largest eddies determines how small the smallest eddies will be compared to them.

In the two-dimensional case considered here the number of degrees of freedom which are necessary to describe the motion is reduced from $R e^{9 / 4}$ to order of $R e$, and thus we have $L / l_{s} \sim R e^{1 / 2}$ (Lesieur, 1987). According to Fig. 4 the first eddies with vertical scale $L \sim 0.05 \mathrm{~m}$ appear when $t=0.3 T$. Taking into account that the value of the particle velocity in the eddies is $U \sim 8 \cdot 10^{-2} \mathrm{~m} \mathrm{~s}^{-1}$ the corresponding Reynolds number $R e$ attains the value $4 \times 10^{3}$. This implies that the smallest scale of expected eddies will be no greater than $l_{s} \sim 10^{-4} \mathrm{~m}$ and, thus, the spatial step of the numerical scheme should not be more than $10^{-4} \mathrm{~m}$. Exactly this value of the spatial grid step was used in our numerical experiments.

\subsection{Regime (ii): solitons}

The "soliton"-scenario covers a substantial part of the parameter space in Fig. 2. Thus, nonlinear internal waves are 
a)

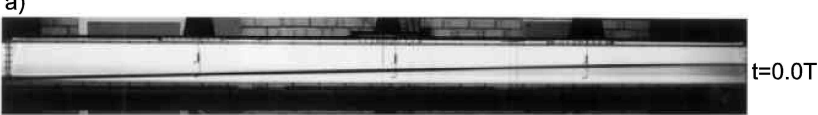

b)
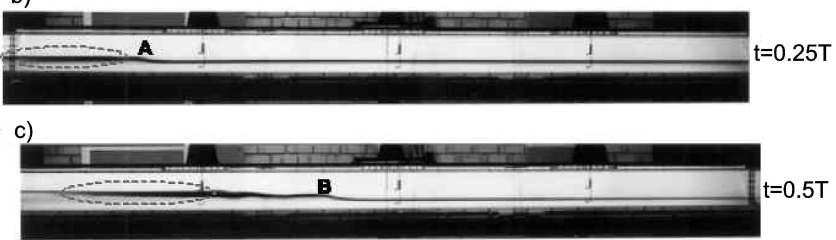

d)

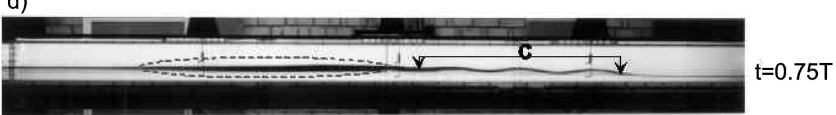

e)

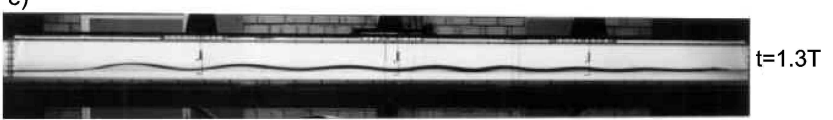

Fig. 6. Time series of photographs showing the steepening of an initial basin-scale wave to form a packet of solitary waves. The widening of the pycnocline is presented by dotted ellipses. Letters A, B and C mark bore, solitary wave and packet of solitary waves (from Horn et al. (2001): reproduced by permission of Cambridge University Press).

expected to be "ordinary" phenomena in the energy cascading from large to small scales.

The time series of one of the experiments presented by Horn et al. (2001) with $h / H=0.3$ and $\eta / h=0.9$ is shown in Fig. 6 (Fig. 4 in Horn et al., 2001). The degeneration of a basin scale internal wave into packets of solitary waves is presented against fractions of the period $T$. Results of Fig. 6 fall into regime (ii), solitary waves and development of internal bores and solitary waves are clearly seen. However, Fig. 6 additionally shows a very interesting phenomenon which were not described and explained by the laboratory experiment. This is the widening of the density interface behind the moving bore to more than double its upstream thickness. The widening has nothing to do with diffusion and mixing because it originates from the beginning of the experiment. Taking into account that the pycnocline widening accompanies the generation of bores and solitary waves, the whole processes of degeneration of internal seiches into short waves deserves investigation.

A time series of numerical modelling with $h / H=0.3$, $\eta / h=0.9$, as used in the laboratory experiment, is presented in Fig. 7. The good agreement between numerical and laboratory results is obvious (compare Figs. 6 and 7). Being initially inclined, the interface begins to return to its equilibrium position. During this stage the horizontal motion of the fluid is accompanied by the vertical motion at the end of the tank. Upwelling of the water at the end of the tank leads to the formation of the internal bore which is clearly seen at the time moment $t=0.25 T$. The solitary wave appears at the instant $t=0.5 T$. Convergence between the position of the solitary wave and the centre of the tank indicates that, very likely, it was formed due to nonlinear processes from
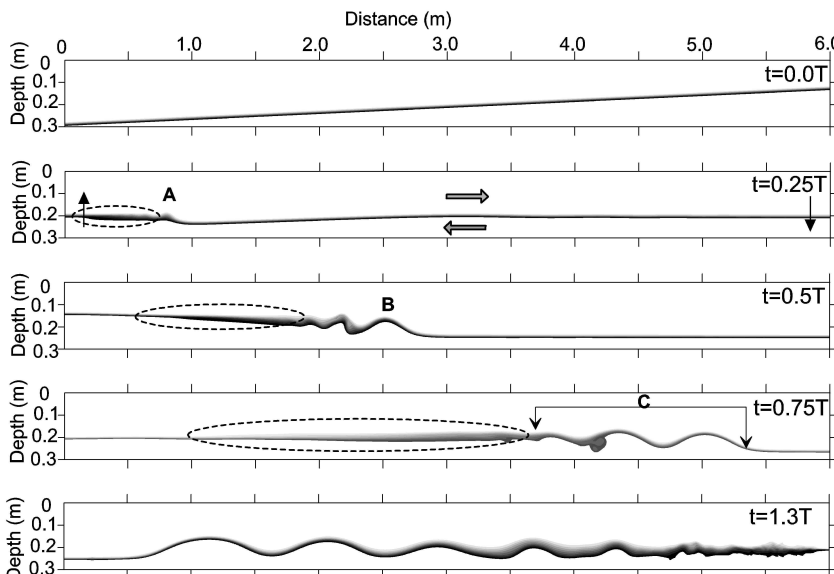

Fig. 7. Time series of case 2 (Fig. 2), the numerical calculations of the density fields for the same time slices as the prototype laboratory tank experiment given in Fig. 6. The widening of the pycnocline is presented by dotted ellipses. Letters A, B and C mark bore, solitary wave and packet of solitary waves.
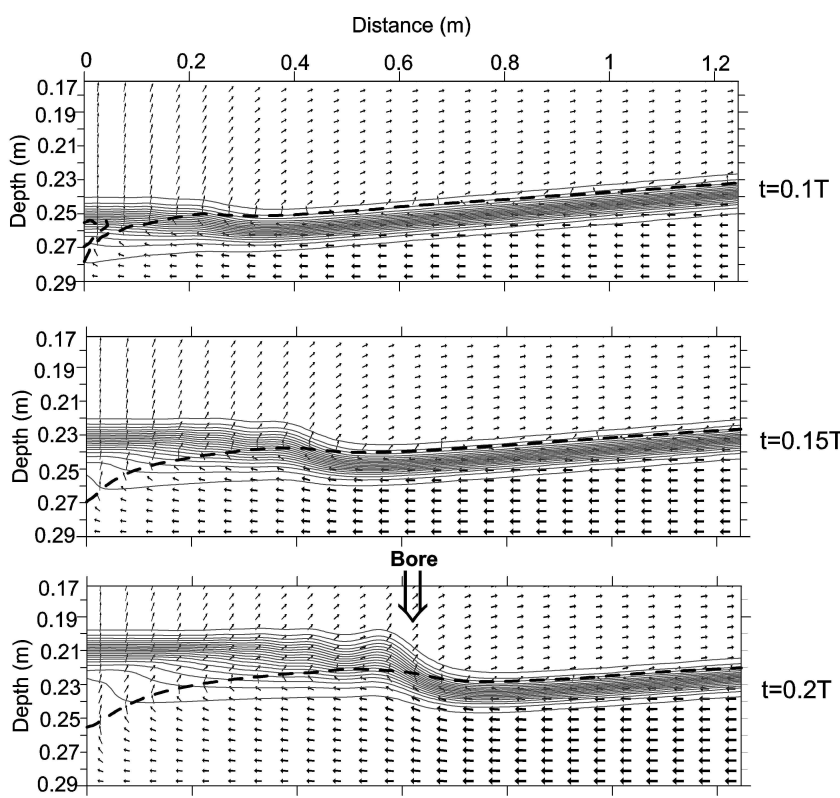

Fig. 8. Snap shots of the density fields (isolines are shown by solid lines), velocity (the magnitude and the direction are given by arrows) and zero isopleth (dashed) near the left wall when $\eta / h=0.9$, $h / H=0.3$ (Figs. 6 and 7).

the bore evolution. The system of solitary waves is also seen at $t=0.75 T$.

The results of the laboratory investigation given in Fig. 6 and the numerical simulation shown in Fig. 7 put forward the question: what is the reason of the pycnocline widening which follows the bore and solitary waves to the opposite side of the tank? The non-hydrostatic model of the continuously stratified fluid takes into account the vertical fluid motion which can be sufficiently large in some parts of the basin and thus give new unexpected results. 

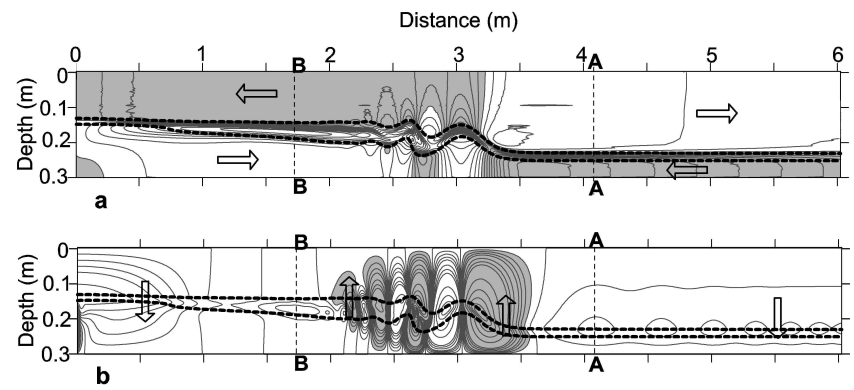

Fig. 9. Isolines of (a) horizontal and (b) vertical velocity fields $(\eta / h=0.9, h / H=0.3)$ at $t=0.5 T$. Grey and white colors correspond to motions in different directions. The position of the isopycnals 1000 and $1020 \mathrm{~kg} \mathrm{~m}^{-3}$ are shown by thick dotted lines. Thick arrows indicate the main direction of the flow.

The formation of the baroclinic wave with the form of a bore, outlined in Figs. 6 and 7, is shown in Fig. 8 in more detail $(\eta / h=0.9, h / H=0.3)$. Here, the velocity fields are superimposed over the density fields. The position of the zero isopleth is given by the dashed line. The basic conclusion, which can be drawn from inspection of Fig. 8, is that the thickness of the density interface close to the wall becomes increased by a factor of two during a very short time (from 0 to $0.2 T$, which equals only $12 \mathrm{~s}$ ). This increase of the thickness of the interface can be explained in terms of the kinematics of a strong wave-wall interaction.

It is obvious that the vanishing of horizontal velocities of the seiche motion can be expected at $T / 2, T, 3 T / 2 \ldots$ Thus the velocities of the generated short internal waves will be predominant in the velocity field in the tank at $t=0.5 T$. Figure 9 shows $u$ and $w$ patterns where opposite currents are shown with a grey-white palette. Isopycnals of 1000 and $1020 \mathrm{~kg} \mathrm{~m}^{-3}$ are given by dashed thick lines.

Further scrutiny of the structure of the solitary waves in the middle of the tank shows that with good accuracy they belong to the first baroclinic mode. The co-phase vertical motions of the pycnocline are here quite evident in the horizontal and vertical velocity fields.

To see these, let us compare the structure of the horizontal velocity field in the front of the leading solitary wave (section AA), and behind the wave train (section BB), where the widening of the pycnocline takes place. Figure 10a displays the vertical structure of $u(z)$ in section AA, and Fig. 10b represents the analogous distribution of the horizontal velocity for section BB. The conclusion to be drawn from these graphs is more than evident. The motion in the front of the wave packet reveals the characteristics of the first baroclinic mode: the surface and bottom layers move in opposite directions, whereas the maximum velocity shear is located in the pycnocline. In addition, the profile $w(z)$ almost ideally fits the profile of the first eigen-function of the standard boundary value problem.

From a first glance one can also conclude that a second mode disturbance (the above mentioned widening of the pycnocline) is moving behind the wave packet. Actually, Fig. 10
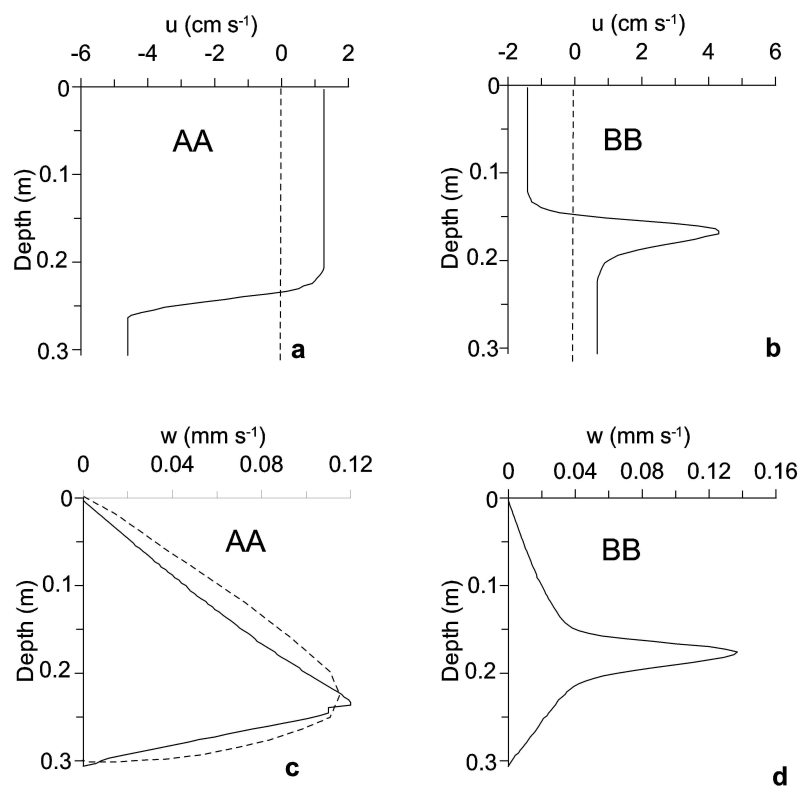

Fig. 10. Dependence with depth of the horizontal (a), (b) and vertical (c), (d) velocity fields obtained for sections AA (a, c) and BB (b, d) from Fig. 9. The dashed profile in (c) shows the first eigenfunction for verical velocity of the standard boundary value problem for vertical density distribution in AA.

shows that the horizontal velocity $u$ possesses a local extremum at the depth of pycnocline which normally correspond to the second baroclinic mode. However, two discrepancies do not support this widening as a propagating secondmode internal wave. The first is that the horizontal motion in the upper and bottom layers must be in phase, whereas Fig. 10 says that this is not the case. Second, the profile of the vertical velocity $w(z)$ in section BB is very different from the structure of the second eigen-function of the standard boundary value problem. The profile $w(z)$ of the second mode should change its sign within the pycnocline layer, whereas in our case it has a maximum value here (Fig. 10d). Thus, the widening of the pycnocline is inconsistent with the assumption that it can be treated as a wave of second baroclinic mode propagating in the tail of the wave packet. More realistically, it is the consequence of advective processes described above and occurring during a strong wave-boundary interaction.

\subsection{Regime (iii): supercritical flow}

If the initial amplitude of the layer inclination $\eta$ is sufficiently large, it may lead to supercritical conditions. Such a case will occur in the tank for $h / H=0.2$ and $\eta / h=1.25$. The supercritical regime applies when the local Froude number (Eq. 5) becomes larger than unity. At the beginning of the motion a turbulent baroclinic bore is formed on the left side of the tank (Fig. 11). This is due to the collision with the faster velocity of denser fluid with the wall than when a typical internal bore in the "solitons" regime (see Fig. 8) is generated. 


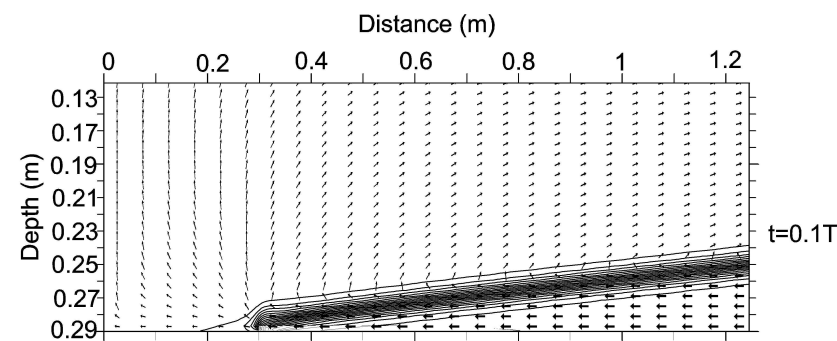

\subsection{Regime (v): supercritical flow and billows}

The regime "supercritical flow and billows" is a combined effect of supercritical flow with large shear in the middle of the basin during the first quarter of the seiche period. This makes the process of wave evolution presented in Fig. 13 more complicated. The difference between these runs and those presented in Fig. 12 is that the ratio of the upper layer thickness to the tank depth is now $h / H=0.3$ instead of 0.2 . Comparison of the upper three panels in Figs. 12 and 13 clearly demonstrates a certain similarity: supercritical flow in the centre of the basin does not allow solitary waves to evolve, as was the case in the "soliton" regime (see also Fig. 7). The only difference between the considered cases is that the amplitude of the initial wave in regime (v) is larger. It suffices to produce strong shear at the interface, which is triggering the generation of billows. They are seen in the middle part of the basin in Fig. 13d behind the leading wave of elevation. These billows provide strong water mixing, seen on the righthand side of the tank in Fig. 13e. Note also that in addition to the enhanced mixing, fast attenuation and energy dissipation takes place in the turbulent area. This circumstance eventually leads to the "soliton" scenario of the flow evolution. As a confirmation, two irregular waves of elevation in Fig. 12e in the middle of the basin can be considered as an initial stage of soliton formation. Generation of billows by strong vertical shear in the central part of the tank is seen even more clearly for yet larger initial amplitudes. Figure $13 \mathrm{f}$ shows the same instant as Fig. 13b, but for the case 5 with $h / H=0.4$ and $\eta / h=1.25$. Evidently, in the last case the flow becomes unstable even earlier, in fact at about $t=0.3 T$. While the growth rate of the Kelvin-Helmholtz instability in case 4 such that it develops with the bore or behind it, Fig. 13f shows that the Kelvin-Helmholtz instability develops much faster at the larger height ratio such that the Kelvin-Helmholtz rolls have grown in advance of the passing of the billow.

\section{Discussion and conclusions}

Figure $12 \mathrm{~b}$ shows the initial stage of the baroclinic turbulent bore formation, whereas panel c represents the well developed supercritical flow. The turbulent baroclinic bore generates vortices which mix water near the interface. When $t=T / 4$, standing seiche oscillations have maximum velocities in the centre of the basin and zero at the ends due to supercritical conditions $(F>1)$ in the middle of the basin but subcritical ones $(F<1)$ at the ends where the standing wave has the maximum vertical displacements. Eventually, as the propagating bore loses its energy due to the generation of turbulence at the interface (including strong interaction with the wall, see, panel d), it evolves into a series of solitary internal waves (Fig. 12e). An example of a rapid change of the background stratification presented in Fig. 3 implies existence of strong water mixing in the interface region. The mixing process in the considered case was also provided by the development of numerous billows, which accompany the water motion.

Energy is supplied to lakes by wind: it drives the surface water and generates internal waves in the form of long-periodic basin-scale standing waves (Mortimer, 1952; Farmer, 1978; Hutter, 1986, 1991). This implies that the internal wave energy in lakes is concentrated in a low-frequency band. At the same time, field experiments indicate that the internal wave field has a continuous spectrum, ranging from these basinscale waves to waves with frequencies approaching the buoyancy frequency maximum (e.g. Thorpe, 1977; Thorpe et al., 1996; Saggio and Imberger, 1998). Thus, the mechanisms that maintain the observed $\sigma^{-2}$ slope of the internal wave spectra in most lakes remain to be identified (here $\sigma$ is the wave frequency).

After storms, internal waves in lakes may take the form of an internal bore or packets of solitons, generated by the nonlinear steepening of a basin-scale finite-amplitude wave (Hunkins and Fliegel, 1973; Thorpe et al., 1972; Thorpe, 


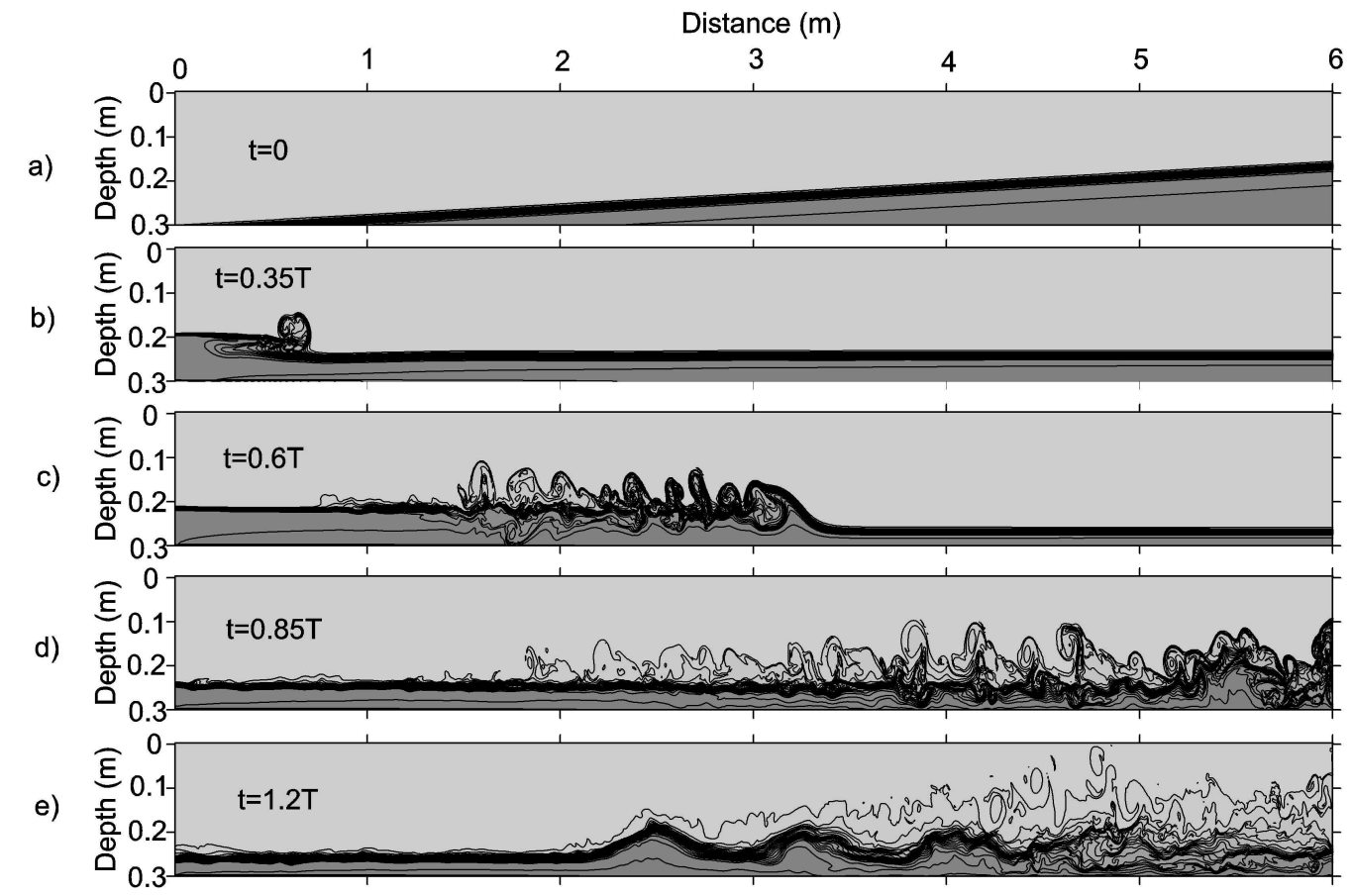

Fig. 12. Time series of density fields for "supercritical flow" regime when $h / H=0.2, \eta / h=1.25$, case 3 (Fig. 2).

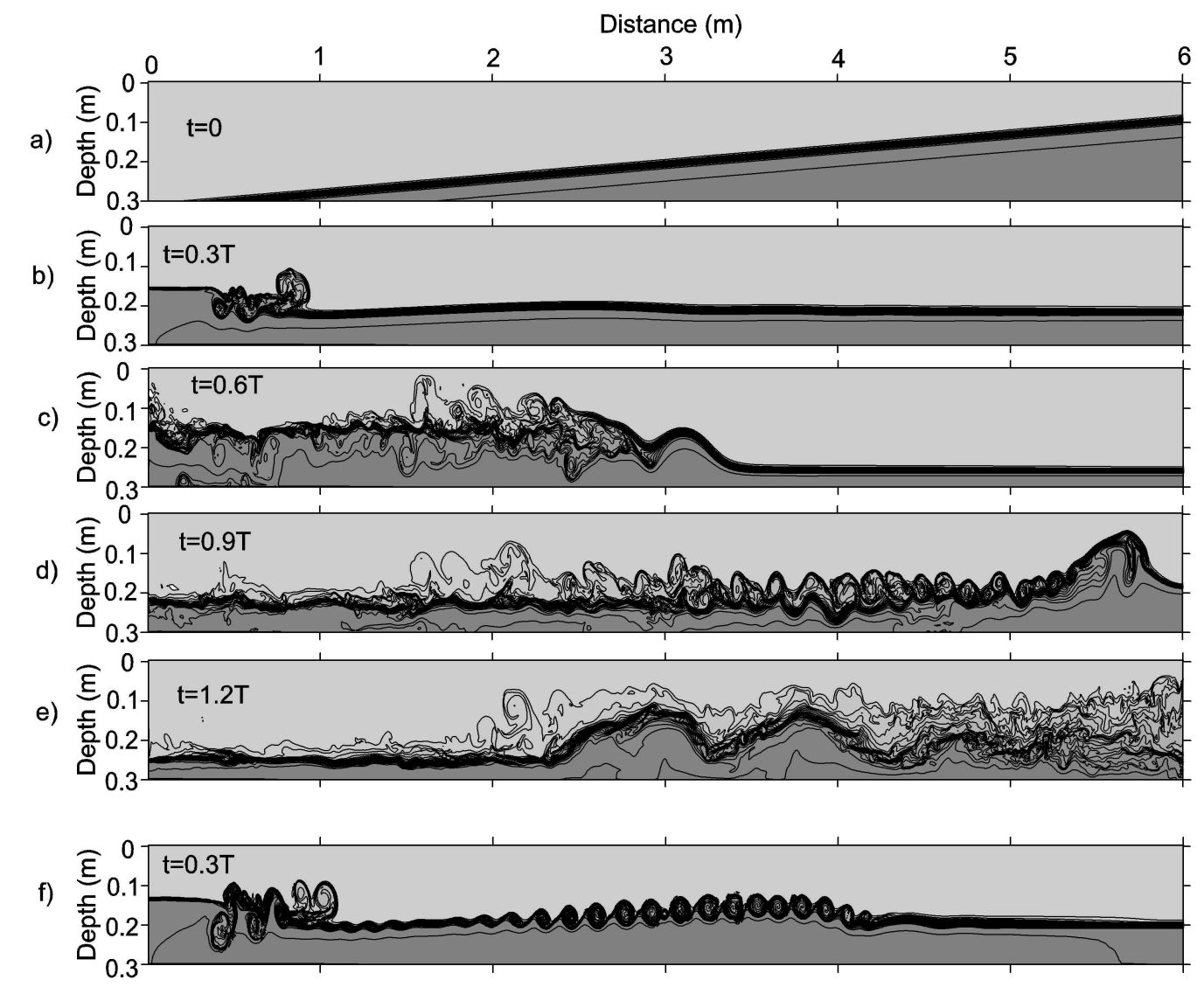

Fig. 13. Regime "supercritical flow and billows": (a)-(e) density field evolution for case 4 (Fig. 2) at $h / H=0.3, \eta / h=1.25$, (f) density field for case 5 (Fig. 2) at $t=0.3 T$ for $h / H=0.4, \eta / h=1.25$. 
1977; Farmer, 1978; Wiegand and Carmack, 1986). Since these solitons are much shorter in length than the windinduced initial large-scale thermocline displacements, their generation results in a transfer of energy within the internal wave field from large to small scales. As a confirmation of such an energy sink, field observations show that windforced basin-scale waves decay at a rate far greater than can be accounted for simply by internal dissipation (Imberger, 1994; Stevens et al., 1996).

An additional evidence of this route for energy transfers from large to small scales was reported by Horn et al. (2001). In this paper, on the basis of field data taken from measurements in long and narrow lakes (viz. Loch Ness, Thorpe et al., 1972 and Babine Lake, Farmer, 1978) it was shown that most of these lakes fall within regime (ii), in which nonlinear steepening predominates, and the disintegration of an initial basin-scale wave occurs as in the example presented in Figs. 6 and 7. Such motion usually originates at conditions of relatively weak wind (with speed $3-4 \mathrm{~m} \mathrm{~s}^{-1}$ ). An important stage of this energy cascading is the formation of solitary waves, which furthermore inevitably encounter lake boundaries, where they are destroyed according to the mechanism of solitary wave breaking described by Vlasenko and Hutter (2002). They showed that the bathymetric structure in the vicinity of lake boundaries strongly affect the structure of the water stratification. In the present study the pycnocline widening is attributed to the interaction with the vertical wall. This effect will become softer when the shore is inclided.

Another scenario of disintegration of a basin-scale wave takes place at larger external impacts. Strong wind increases the amplitude of baroclinic seiches so that the latter generates seiche-induced hydrodynamic instabilities (see as an example Figs. 3, 12 and 13). Further evolution is characterised by formation of billows, their successive disintegration into turbulent patches, which, finally, attenuate and form new background stratification. This process was reported, for instance, in the laboratory experiments by Thorpe $(1971,1973)$. For such regimes we can expect considerable mixing and remarkable changes of the pycnocline structure for the entire basin.

Acknowledgements. This work was funded by INTAS project 03-51-3728. We thank the anonymous reviewers and W.-G. Früh (editor), whose comments have led to significant improvement of the paper.

Edited by: W.-G. Früh

Reviewed by: two referee

\section{References}

Batchelor, G. K.: An introduction to fluid dynamics, Cambridge University Press, 615 pp., 1967.

Farmer, D. M.: Observations of long nonlinear waves in a lake, J. Phys. Oceanogr., 8, 63-73, 1978.
Horn, D. A., Imberger, J., and Ivey, G. N.: The degeneration of large-scale interfacial gravity waves in lakes, J. Fluid Mech., 434, 181-207, 2001.

Hunkins, K. and Fliegel, M.: Internal undular surges in Seneca Lake: A natural occurrence of solitons, J. Geoph. Res., 78, 539548, 1973.

Hutter, K.: Hydrodynamic modeling of lakes, in: Encyclopedia of fluid mechanics, edited by: Cheremisinoff, N. P., Gulf Publishing Company, Houston, 6, 289-403, 1986.

Hutter, K. (with contributions by Bäuerle, E., Salvadé, G., Spinedi, C., and Zamboni, I.): Large scale water movements in lakes, Aquatic Sciences, 53, 100-135, 1991.

Imberger, J.: Transport processes in lakes: A review, in: Limnology now: A paradigm of planetary problems, edited by: Margalef, R., Elsevier, 99-193, 1994.

Kolmogorov, A. N.: The local structure of turbulence in incompressible viscous fluid for very large Reynolds numbers. Dokl. Akad. Nauk SSSR, 30, 301-305, 1941.

Lesieur, M.: Turbulence in fluids. Stochastic and numerical modelling, Martinus Nijhoff Publishers, 286 pp., 1987.

Mortimer, C. H.: Water movements in lakes during summer stratification: Evidence from the temperature distribution in Windermere, Phil. Trans. R. Soc., London, B236, 255-404, 1952.

Ostrovsky, L. A. and Stepanynts, Yu. A.: Do internal solitons exist in the ocean?, Rev. Geophys., 27, 293-310, 1989.

Saggio, A. and Imberger, J.: Internal wave weather in a stratified lake, Limnol. Oceanogr., 43, 1780-1795, 1998.

Stevens, C.: Internal waves in small reservoir, J. Geophys. Res., 104, 15 777-15 788, 1999.

Stevens C., Lawrence, C., Hamblin, P., and Imberger, J.: Wind forcing of internal waves in a long stratified lake, Dyn. Atmos. Oceans, 24, 41-50, 1996.

Thorpe, S. A.: Experiments on the instability of stratified shear flow: miscible fluids, J. Fluid Mech., 46, 299-319, 1971.

Thorpe, S. A.: Experiments on the instability and turbulence in a stratified shear flow, J. Fluid Mech., 61, 731-751, 1973.

Thorpe, S. A.: Evidence of Kelvin-Helmholtz billows in Loch Ness, Limnology and Oceanogrphy, 19, 973-976, 1974.

Thorpe, S. A., Hall, A. J., and Croft, I.: The internal surge in Loch Ness, Nature, 237, 96-98, 1972.

Thorpe, S. A.: Turbulence and mixing in a Scottish loch, Phil. Trans. R. Soc. Lond., A286, 125-181, 1977.

Thorpe, S. A., Keen, J. M., Jiang, R., and Lemmin, U.: Highfrequency internal waves in Lake Geneva, Phil. Trans. R. Soc. Lond., A354, 237-257, 1996.

Turner, J. S.: Buoyancy effects in fluids, Cambridge University Press, 367 pp., 1973.

Vlasenko, V. and Hutter, K.: Transformation and disintegration of strongly nonlinear internal waves by topography in stratified lakes, Ann. Geophys., 20, 2087-2103, 2002,

SRef-ID: 1432-0576/ag/2002-20-2087.

Vlasenko, V., Stashchuk, N., and Hutter, K:. Baroclinic tides: theoretical modeling and observational evidence, Cambridge University Press, 351 pp., 2005.

Wiegand, R. C. and Carmack, E.: The climatology of internal waves in a deep temperature lake, J. Geoph. Res., 91, 3951-3958, 1986. 